\title{
A Model to Determine Naked-Eye Limiting Magnitude
}

\author{
Dasha Crocker ${ }^{1}$, Vincent Schmidt ${ }^{1}$ and Laura Schmidt ${ }^{1}$ \\ ${ }^{1}$ Bellbrook High School, Bellbrook, OH, USA
}

\section{$\underline{\text { ABSTRACT }}$}

The purpose of this study was to determine which variables would be needed to generate a model that predicted the naked eye limiting magnitude on a given night. After background research was conducted, it seemed most likely that wind speed, air quality, skyglow, and cloud cover would contribute to the proposed model. This hypothesis was tested by obtaining local weather data, then determining the naked eye limiting magnitude for the local conditions. This procedure was repeated for the moon cycle of October, then repeated an additional 11 times in November, December, and January. After the initial 30 trials, $r$ values were calculated for each variable that was measured. These values revealed that wind was not at all correlated with the naked eye limiting magnitude, but pollen (a measure of air quality), skyglow, and cloud cover were. After the generation of several models using multiple regression tests, air quality also proved not to affect the naked eye limiting magnitude. It was concluded that skyglow and cloud cover would contribute to a model that predicts naked eye limiting magnitude, proving the original hypothesis to be partially correct.

\section{Introduction}

Humanity has been looking to the stars for thousands of years. Ancient civilizations looked to the stars hoping that they could explain the world around them. Mayans invented shadow casting devices to track the movement of the sun, moon, and planets, while Chinese astronomers discovered Ganymede, one of Jupiter's moons (Cook, 2018). Today, astronomers use complex observation techniques to learn more about our solar system and beyond.

Professional astronomers have made many discoveries with telescopes of all kinds. Orbiting telescopes have discovered new stars, taken photos of distant galaxies, and detected possible threats to Earth. Even with these telescopes, it is still necessary to use ground-based observatories because orbital telescopes are sometimes impractical. At these locations, local viewing conditions can have a large impact on observations. Before these conditions can be understood, it is first necessary to understand the vocabulary astronomers use to describe the sky.

\section{Understanding Magnitude}

In astronomy, magnitude refers to how bright or dim a star appears in the sky. Stars with a smaller magnitude are brighter than stars with a greater one. This scale is also logarithmic, meaning that a $1^{\text {st }}$ magnitude is approximately 2.5 times brighter than a $2^{\text {nd }}$ magnitude star, and a $2^{\text {nd }}$ magnitude star is 2.5 times brighter than a $3^{\text {rd }}$ magnitude star (McClure, 2017). There are two types of magnitude: absolute magnitude and apparent magnitude. Apparent magnitude refers to how bright the star is when seen from Earth. The absolute magnitude will not be discussed in this study (Apparent and Absolute Magnitudes, 1998).

In a rural area with optimal viewing conditions, a human can see a magnitude 6 star with the naked eye. In suburban areas, this limit drops to magnitude 3 , and only the brightest stars can be seen in urban areas. With binoculars, a human can see a magnitude 9.5 star. The Hubble space telescope, however, can observe a magnitude 30 star. (McClure, 2017). 


\section{Viewing Scales}

Astronomers have already created scales that can be used to determine general viewing conditions. These scales date back to the $19^{\text {th }}$ century when astronomers used words like "perfect" or "terrible" to describe the night sky. Because these observations were very subjective, astronomers wanted to quantify their observations of the night sky in order to communicate their results more effectively (MacEvoy, 2013).

The Antoniadi scale was developed in 1909 to standardize viewing scales. Still widely used today, this scale rates viewing conditions from I (perfect seeing, without a quiver) to V ( very bad seeing, scarcely allowing the drawing of a rough sketch). Astronomers prefer to use the middle three values on this scale, as the descriptions for the ends of this scale are extreme. This scale is largely subjective, as it relies on verbal descriptions (Curtis, 1969).

The Bortle dark-sky scale uses levels of skyglow to determine what stars are able to be seen. This scale is rated on a 1 to 9 scale, and uses factors like limiting magnitude, the dimmest star that can be seen with the naked eye, and the visibility of certain landmarks to determine what class the sky falls into. This scale also has subjective descriptions such as "entire sky is washed" or "sky is completely dark." The Bortle scale, while it does provide quantitative measurements of star visibility, it only uses skyglow to determine what a person will be able to see on a given night (John Bortle's Light Pollution Scale, 2017). Astronomers believe that many factors will affect viewing conditions, but some factors may have a greater effect on viewing conditions.

\section{Factors That May Affect Viewing Magnitude}

The dimmest observable star, or limiting magnitude, can inform astronomers about the overall quality of the sky. If a higher magnitude star is seen, an astronomer has access to a wider range of observable objects (John Bortle's Light Pollution Scale, 2017). Professional astronomers use large diameter telescopes, which capture more light from the sky in order to observe dimmer objects in the sky, while casual observers may rely on their own eyes to observe the sky. In both situations, many factors may affect an observer's ability to view dimmer stars.

Cloud cover is most associated with the ability to view stars. Astronomers and weather forecasters use the terms such as clear and cloudy to describe cloud cover, but what do these mean? The term "clear" means that none of the skies is covered with opaque clouds. "Mostly clear" means that $12.5 \%$ to $25 \%$ of the sky is covered with clouds and "partly cloudy" means that $37.5 \%$ to $62.5 \%$ of the sky is covered by clouds. A sky that is "mostly cloudy" is $75 \%$ to $87.5 \%$ covered by clouds, and a "cloudy" sky is one that is $100 \%$ covered by opaque clouds (Soniak, 2017).

Skyglow is also frequently associated with the ability to view certain stars (Cinzano, 2001). Skyglow is light from artificial sources that is reflected into the sky and is then reflected back to Earth by particulate matter in the atmosphere. Skyglow can be measured using the Bortles scale, which rates skies from 1 to 9, where 1 is a dark sky, and 9 is an inner-city sky. Another measure of skyglow is magnitude per arcsecond, which gives a number that shows how bright the sky would be if a star of the given magnitude were spaced across the sky every 1 arcsecond (International Dark-Sky Association, 2019b).

Astronomers believe that wind, particularly in the upper atmosphere, can also affect star visibility. These winds increase the amount of atmosphere that obscures stars, sometimes making dimmer ones completely invisible to the naked eye. Winds in the upper atmosphere also combine hot and cool air, each of which refracts light at different angles. This results in the light from dim stars being scattered, thus rendering these stars invisible. Turbulence in the atmosphere can also increase how much a star 'twinkles' (John Bortle's Light Pollution Scale, 2017).

Air quality is a measure of particulate matter in the atmosphere. The pollen index measures pollen in the air and puts it on a 1 to 12 scale, where 1 is low and 12 is high. The air quality index (AQI) measures pollen as well as other pollutants and returns a value from 1 to 500, where 1 is low and 500 is high. Astronomers generally believe that particulate matter affects viewing, but have not yet used these indexes to determine viewing ability (Hodge, 1972).

Humidity is a measure of how much water is in the air relative to how much water the air can hold. It can affect a person's ability to view stars, particularly through telescopes. In humid conditions, telescope lenses can fog 
up, making it difficult to view stars. High humidity means that there is a high concentration of water, which refracts light, in the air, so astronomers believe that humidity may also have some effect on visual observing as well (Woods, 2015). Dew point is also related to humidity, measuring the temperature at which water in the air will condense (US Department of Commerce, 2019).

Temperature is a measure of average kinetic energy in a given system. Although sudden changes in temperature will result in a haze near the horizon, this measure is unlikely to affect observing conditions significantly. Air pressure, which is closely related to temperature, is a measurement that determines the force per unit area at a given location. High-pressure systems are created when wind currents flow out of the system, and low-pressure systems are created when air flows into the system. Because pressure is related to temperature, this measure is unlikely to affect viewing conditions (MacRobert, 2017).

The UV index measures the amount of UV radiation in the atmosphere. Since this measure is almost solely caused by the sun, it is extremely unlikely that this measure will affect viewing conditions (UV Index, 2019).

Visibility is a measure of the distance that an object can be identified from against a background on an undisrupted horizon. Particulate matter, fog, and other pollutants all affect visibility. This measure is likely to affect viewing conditions, because, although astronomers do not specifically mention this measure as something that might affect viewing conditions, visibility is a combination of factors that are likely to affect viewing conditions (Hodge, 1972).

As seen above, there are many factors that may affect the viewing conditions on a given night. Although astronomers believe that only cloud cover, skyglow, air quality, and wind will have a significant effect on viewing conditions, it seems necessary to determine whether other factors, such as humidity, visibility, or temperature will also affect viewing conditions.

\section{Methods}

This research was conducted in one area of the sky. This area, referred to as the observation area, included circumpolar stars and stars that comprise Ursa Major. This area was chosen because circumpolar stars are visible all night and at all times of the year in the northern hemisphere. Ursa Major was included because it is one of the most recognizable constellations in the sky. Stars in this region were used to determine the naked-eye limiting magnitude during each observation (Ford, 2019).

Data were collected at approximately $9 \mathrm{pm}$ Daylight Savings Time every night for the moon phase of October 2019, from new moon to new moon. Weather parameters included moon phase, cloud cover, dew point, humidity, temperature, pollen index, air quality index, pressure, visibility, wind speed, and UV index. The following procedures were executed each observation night. The researcher drove to the observation location around 9 pm DST, 'weather' parameters were recorded, and the dimmest magnitude star seen with the naked eye during a 10-minute window was also recorded. The magnitudes of stars are well-defined, so the dimmest star seen was used to approximate naked-eye limiting magnitude. To minimize bias introduced by the researcher, the following steps were executed during observations:

The researcher adjusted to the dark in the fifteen minutes prior to the observation period. This included using blue light filters on electronic devices. During the observation period, the researcher confirmed the naked-eye limiting magnitude by locating the dimmest star seen twice. This aimed to reduce any bias produced by knowing the locations of each star.

The researcher also aimed to reduce bias by recording skyglow measurements after the dimmest star was seen. Knowing the exact skyglow levels may have led the researcher to believe they had seen a dimmer star than they actually had. Skyglow measurements were taken using the Dark Sky Meter phone app available only on the app store according to recommendations from the International Dark Sky Association (International Dark Sky Association, 2019a). Three locations were used to collect data. Location 1 was used as a primary location, however locations 2 and 3 were used when location 1 could not be used. 


\section{Location 1: Walton Park}

Description: The night sky is fairly crisp on a cloudless night, although a haze from a nearby town to the North makes it difficult to see stars that are low in the sky. There are also lights in the field, but they are located behind the observer when facing North.

Weather Underground station: Waynesville station

\section{Location 2: Veteran's Memorial Park in Morrow, Ohio}

Description: This location is closer to Morrow, but there is less haze on the horizon, probably due to the use of nonLED streetlights. There are lights towards the northern sky, but they do not obscure the night sky noticeably.

Weather Underground station: Morrow station

\section{Location 3: Woodland Trails Scout Camp in Camden, Ohio}

Description: Being very comparatively far away from Camden, this location has almost no haze near the horizon. There is a bright light behind the observer that obscures the stars closer to the horizon.

Weather Underground station: Camden station.

For the purposes of the experiment, skyglow included any light that obscures stars seen in the night sky. This definition includes natural light sources and non-natural ones, whereas the traditional definition only includes humanmade light sources. This study measured skyglow using magnitude per arcsecond. More data points were included subsequently, time and weather permitting, in order to increase the sample size.

After data were collected, predictive models were generated using multiple regression tests. To determine which variables would be included in each model, Ordinary Least Squares regressions were used to show which variables were most correlated with the naked-eye limiting magnitude. $\mathrm{R}$ values produced by these tests were indicators of whether certain variables would be included and not intended to serve as predictors alone.

\section{Results}

Thirty observations were made during the moon phase of October 2019. Six of these observations did not include the limiting magnitude, the dependent variable in this study, because a star was not found during these observations. Because a star could not be seen, these observations were excluded from the dataset because a multiple regression requires that all data be present for analysis. Eleven additional observations were made during the months of November and December 2019 as well as January 2020.

\section{Simple Regression Tests}

Each parameter was compared to the limiting magnitude using a simple linear regression in order to determine which variables would be included in future models. Cloud cover ( $\mathrm{r}=0.70)$, skyglow $(\mathrm{r}=0.56)$, pollen index $(\mathrm{r}=0.57)$, air quality index $(\mathrm{r}=0.36)$ and temperature $(\mathrm{r}=0.37)$ were most correlated with limiting magnitude. Although predicted to be somewhat correlated with limiting magnitude, wind speed only had an r-value of 0.21 . Visibility, although somewhat correlated with limiting magnitude, did not show variance and could not be used to accurately predict limiting magnitude. (Table 1). 
Table 1: R-Values

\begin{tabular}{|c|c|c|c|c|}
\hline & Time & Moon illumination (\%) & Cloud Cover & Skyglow \\
\hline R-Value & 0.08 & 0.06 & 0.70 & 0.56 \\
\hline & Temperature & Pollen Index & Air Quality Index & Pressure \\
\hline R-Value & 0.37 & 0.57 & 0.36 & 0.14 \\
\hline & Humidity & Visibility & Dew Point & Wind Speed \\
\hline R-Value & 0.02 & $0.35^{*}$ & 0.36 & 0.21 \\
\hline
\end{tabular}

\section{Predictive Models}

Multiple regression statistical analyses were used to predict the naked-eye limiting magnitude. Variance Inflation Factor tests showed that little multicollinearity existed between the variables highly correlated with naked-eye limiting magnitude. (Table 2).

Table 2: VIF Values

\begin{tabular}{|c|c|c|c|c|}
\hline Variable 1 & Cloud Cover & Cloud Cover & Cloud Cover & Cloud Cover \\
\hline Variable 2 & Skyglow & Pollen Index & Temperature & Air Quality Index \\
\hline VIF & 1.16 & 1.24 & 1.01 & 1.01 \\
\hline Variable 1 & Skyglow & Pollen Index & Pollen Index \\
\hline Variable 2 & Air Quality Index & Temperature & Air Quality Index \\
\hline VIF & 1.00 & 1.01 & 1.00 \\
\hline Variable 1 & Temperature & Temperature & Skyglow \\
\hline Variable 2 & Air Quality Index & 1.00 & 1.00 \\
\hline VIF & 1.13 & \\
\hline
\end{tabular}

Based on the results of each simple regression, variables most highly correlated with limiting magnitude were used as factors in multiple regression analyses. After several iterations of predictive models were generated, only cloud cover and skyglow had a significant effect on naked-eye limiting magnitude. Each iteration was tested for outliers using the residuals of each given model. A value was only excluded when it was found to be an outlier in three separate models to compensate for possible variation between models.

The final model used cloud cover and skyglow to predict the naked-eye limiting magnitude and included 33 trials. This significant model $(\mathrm{p}=1.41 \times 10-11)$ predicted $86 \%$ of the variance in limiting magnitude. Cloud cover was not included as a continuous variable, but as a categorical variable because cloud cover is typically reported as a categorical variable. The categories used to report cloud cover were clear, mostly clear, partly cloudy, and mostly cloudy.

Table 3: Summary of Predictive Model Results

\begin{tabular}{|c|c|c|c|c|c|}
\hline Multiple R & R Squared & Standard Error & F & Significance F & Observations \\
\hline 0.928 & 0.860 & 0.312 & 43.1 & $1.41 \times 10^{-11}$ & 33 \\
\hline Factors & & \multicolumn{3}{c|}{ Coefficients } & P-Value \\
\hline Skyglow $\left(\mathrm{X}_{1}\right)$ & 0.580 & $5.76 \times 10^{-5}$ \\
\hline Clear & 1.93 & $2.53 \times 10^{-9}$ \\
\hline \\
Mostly Clear & 2.00 & $1.19 \times 10^{-6}$ \\
\hline
\end{tabular}


This predictive model was defined by four separate linear equations whose slopes are defined by the variable skyglow. The y-intercepts of each line were predicted by the cloud variable. While the predictive line for "Mostly Clear" is above the line for "Clear", this difference is not significant and likely due to an insufficient quantity of data. (Figure $1)$.

\section{Figure 1: Predictive Model Equations}

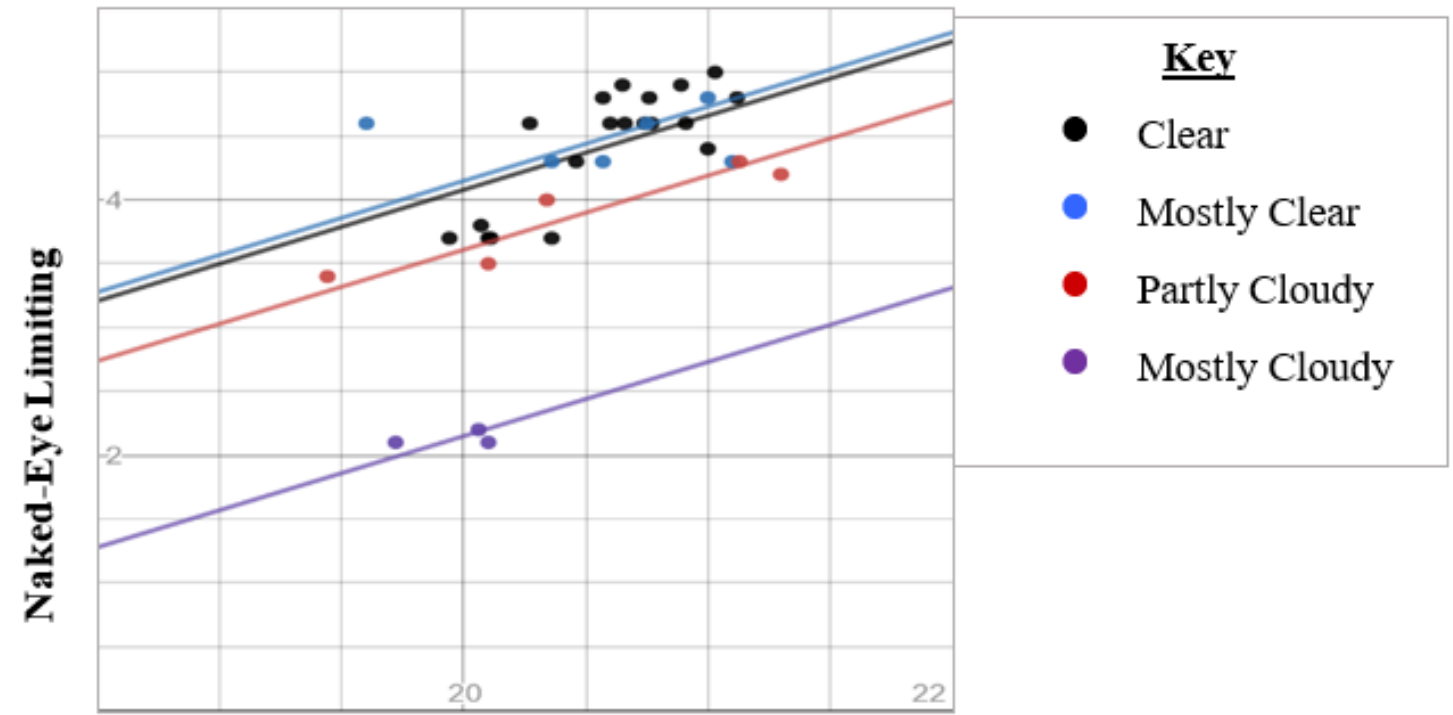

Skyglow

\section{Discussion}

The goal of this study was to determine what factors affect the naked eye limiting magnitude on a given night. It was determined that cloud cover and skyglow have a significant effect on the naked eye limiting magnitude. This is contrary to the hypothesis that wind speed and the air quality index would also affect the naked eye limiting magnitude. It seems unlikely that wind will determine if a certain star can be seen, but it may determine how well a star can be seen (how much the star blurs when observed), this factor was excluded from this study. Based on these results, the air quality index seems unlikely to affect the naked eye limiting magnitude for a given night, but the values recorded on this scale stayed below 80 when the scale reaches a maximum of 500. It may be possible that air quality may affect the limiting magnitude if it reaches a higher value.

The other goal of this study was to generate models that could predict the limiting magnitude using the least number of variables possible. While early predictive models mostly succeeded, even the best of these models was flawed and used many variables. While the $\mathrm{R}$ values of these models were comparable to the $\mathrm{R}$-value of the final model, the early models contained more variables, meaning that the greater $\mathrm{R}$ values were due to the greater number of variables. Additional observations were made to increase the statistical validity of early models. The final predictive model was more predictive than early models and only used cloud cover and skyglow to make predictions.

\section{Implications for Further Research}

This study showed that a model could be generated to determine the naked eye limiting magnitude. A further study could determine whether this model could be adjusted for different sizes of telescopes, or new models would need to 
be generated. A second study could standardize the model that was created for all types of locations and most people. This study could use multiple participants from different locations. This study would account for potential human bias, so the predictive models it would generate could be more widely used A program could also be written to generate a model for a particular individual based on viewing conditions in the area. More data could be collected in order to generate a model for each season of the year.

\section{Conclusion}

The models generated from this study showed that a model can be generated to predict the naked eye limiting magnitude based on local viewing conditions. These models differed from other viewing scales (such as the Antoniadi scale and the Bortle Dark Sky scale) by providing the naked eye limiting magnitude based on multiple objective factors instead of subjective observations. Astronomers currently use subjective scales to determine when to observe but using quantitative factors will allow astronomers to better determine when to observe, which will save time and money. Astronomers can use the model generated from this study to quickly predict the approximate naked-eye limiting magnitude. The variables in the final model that predicted the naked eye limiting magnitude were cloud cover and skyglow, although the hypothesis of this study predicted that wind speed and the air quality index would also be included in the final model. Based on these results, the original hypothesis of this study is partially correct.

\section{Acknowledgments}

Conan, Camon and Alison Crocker drove out to Walton Park to assist in data collection. Alison, Conan, Edith and Halie Crocker checked the written portions for grammatical errors. Vincent and Laura Schmidt served as mentors for this project. Thanks to everyone who made this project possible.

\section{Works Cited}

Apparent and Absolute Magnitudes. (1998). Retrieved September 29, 2019, from http://www.phys.ksu.edu/personal/wysin/astro/magnitudes.html

Cinzano, P., Falchi, F., \& Elvidge, C. D. (2001). Naked-eye star visibility and limiting magnitude mapped from DMSP-OLS satellite data. Monthly Notices of the Royal Astronomical Society, 323(1), 34-46. doi: 10.1046/j.13658711.2001.04213.x

Cook, J. (2018). 7 Ancient Cultures and How They Shaped Astronomy. Retrieved September 29, 2019, from http://www.astro4dev.org/blog/2018/11/26/7-ancient-cultures-and-how-they-shaped-astronomy-guest-blog/

Curtis, A. C. (1969). Seeing Conditions: A Plea (Antoniadi Scale). The Astronomer, 6, 21-22.

Retrieved from http://adsabs.harvard.edu/full/1969Astr...6...21C.

Ford, D. (2019). The Constellation Ursa Major. Retrieved September 20, 2019, from https://in- the-sky.org/data/constellation.php?id=85

Hodge, P. W., Laulainen, N., \& Charlson, R. J. (1972, December 8). Astronomy and air pollution. Retrieved September 29, 2019, from http://www.ncbi.nlm.nih.gov/pubmed /17741988. 
International Dark Sky Association. (2019a, April 22). How To Conduct A Sky Quality Survey. Retrieved September 29, 2019, from http://www.darksky.org/our-work/conservation/ idsp/become-a-dark-sky-place/sky-quality-survey/

International Dark Sky Association. (2019b, May 3). Measuring Light Pollution. Retrieved September 29, 2019, from http://www.darksky.org/light-pollution/measuring-light-pollution/

John Bortle's Light Pollution Scale. (2017, January 20). Retrieved October 22, 2019, from https://www.novac.com/wp/observing/bortle-scale/

MacEvoy, B. (2013). Astronomical Seeing. Retrieved September 29, 2019, from http://www.handprint.com/ASTRO/seeing2.html

MacRobert, A. (2017, May 8). How to Successfully Beat Atmospheric Seeing. Retrieved October 22, 2019, from http://www.skyandtelescope.com/astronomy-equipment/beating-the-seeing/

McClure, B. (2017). What is stellar magnitude? Retrieved October 23, 2019, from https://earthsky.org/astronomyessentials/what-is-stellar-magnitude

Soniak, M. (2017, June 27). What's the Difference Between "Mostly Sunny" and "Partly Cloudy"? Retrieved September 29, 2019, from http://www.mentalfloss.com/article/ 56820/whats-difference-between-mostly-sunny-andpartly-cloudy

US Department of Commerce, \& Noaa. (2019, June 18). Dew Point vs Humidity. Retrieved October 22, 2019, from http://www.weather.gov/arx/why dewpoint vs humidity

UV Index. (2019, May 13). Retrieved 28, 2019, from http://www.epa.gov/sunsafety/uv-index-1 Waynesville, OH Weather Conditionsstar_ratehome. (2019, September 28). Retrieved September 28, 2019, from http://www.wunderground.com/weather/us/oh/waynesville/KOHWAY NE16? utm source=HomeCard\&utm content=Button\&cm ven=HomeCardButton.

Woods, C. (2015, July 31). 8 things you didn't know about humidity. Retrieved October 22, 2019, from http://www.pbs.org/newshour/science/8-things-didnt-know-humidity 